\title{
PERIODIC SOLUTIONS OF NON-DENSELY DEFINED DELAY EVOLUTION EQUATIONS
}

\author{
KHALIL EZZINBI \\ Faculty des Sciences Semlalia \\ Departement de Mathematiques, BP 2390 \\ Marrakech, Morocco \\ JAMES H. LIU \\ James Madison University \\ Department of Mathematics \\ Harrisonburg, VA 22807 USA
}

(Received July, 2000; Revised May, 2001)

We study the finite delay evolution equation

$$
\left\{\begin{array}{l}
x^{\prime}(t)=A x(t)+F\left(t, x_{t}\right), \quad t \geq 0, \\
x_{0}=\varphi \in C([-r, 0], E),
\end{array}\right.
$$

where the linear operator $A$ is non-densely defined and satisfies the Hille-Yosida condition. First, we obtain some properties of "integral solutions" for this case and prove the compactness of an operator determined by integral solutions. This allows us to apply Horn's fixed point theorem to prove the existence of periodic integral solutions when integral solutions are bounded and ultimately bounded. This extends the study of periodic solutions for densely defined operators to the non-densely defined operators. An example is given.

Key words: Periodic Solutions, Non-Densely Defined Equations.

AMS subject classifications: 34G.

\section{Introduction}

Herein, we consider finite delay evolution equation

$$
\left\{\begin{array}{l}
x^{\prime}(t)=A x(t)+F\left(t, x_{t}\right), \quad t \geq 0, \\
x_{0}=\varphi \in C([-r, 0], E),
\end{array}\right.
$$

where $A$ is a non-densely defined linear operator in a Banach space $E ; C=C([-r, 0], E)$ is the space of continuous functions from $[-r, 0](r>0$ is a constant) to $E$ endowed with the super-norm; and for every $t \geq 0$, the function $x_{t} \in C$ is defined by

$$
x_{t}(\cdot)(\theta)=x_{t}(\theta)=x(t+\theta), \quad \theta \in[-r, 0],
$$

and $F(t, \varphi)$ is a continuous function from $\mathbb{R} \times C$ to $E$, and is $\omega$-periodic in $t$. 
It is well known that if $A$ is the infinitesimal generator of a $C_{0}$-semigroup of bounded linear operators, or equivalently,

(i) $\overline{D(A)}=E,(D$ means domain),

(ii) there exist $M \geq 0$ and $\tau \in \mathbb{R}$ such that $] \tau, \infty\left[\subset \rho(A)\right.$, and $\sup \left\{(\lambda-\tau)^{n}\right.$ $\left.\left|(\lambda-A)^{-n}\right|: \lambda>\tau, n \in N\right\} \leq M$

where $\rho(A)$ is the resolvent set of $A$, then the classical semigroup theory ensures the wellposedness of Equation (1.1) [12].

In this work, we investigate the case when the operator $A$ satisfies only the assumption (ii), that is, when $A$ is non-densely defined and we are concerned with the existence of periodic solutions for Equation (1.1). Related studies and examples concerning non-densely defined operators can be found in references such as [1, 3, 4, 7]. For example, in [3], the authors prove that Equation (1.1) is well-posed in the set

$$
C_{0}=\{\varphi \in C([-r, 0], E): \varphi(0) \in \overline{D(A)}\} .
$$

The problem of finding periodic solutions is an important subject in the qualitative study of ordinary and functional differential equations. The famous Massera's theorem [9] on twodimensional periodic ordinary differential equations explains the relationship between the bounded solutions and periodic solutions. Using Browder's fixed point theorem, Yoshizawa proved in [13] that, if the solutions of an $n$-dimensional periodic ordinary differential equation are either uniformly bounded or uniformly ultimately bounded, then the system has a periodic solution. Hale and Lopes [6] used Horn's fixed point theorem to obtain the same result for $n$-dimensional periodic ordinary and functional differential equations with finite delay. In [11], Show studies Massera's theorem for some functional differential equations in finite dimensional spaces and proves that the existence of a bounded solution implies the existence of a periodic solution.

In may of those studies, the most important feature is to show that the operator

$$
P \phi=x_{\omega}(\cdot, \phi), \quad(\omega \text { units along } x)
$$

is compact (continuous and takes a bounded set into a precompact set), where $\omega$ is the period of the system and $x$ is the unique solution determined by $\phi$. Then, some fixed point theorem can be used to derive periodic solutions.

For equations in general Banach spaces (infinite dimensional), showing the compactness of operator $P$ is a very hard task due to the difficulty involving the abstract version of ArzelaAscoli's theorem. As can be seen in [2, 8, 14], if this difficulty can be overcome, then other steps for equations in finite dimensional spaces can be carried over to show that operator $P$ is compact, and hence to derive periodic solutions. For example, in [8], the author shows the compactness of $P$ for the evolution equation

$$
\left\{\begin{array}{l}
x^{\prime}(t)=A(t) x(t)+f\left(t, x(t), x_{t}\right), \quad t \geq 0 \\
x_{0}=\varphi \in C([-r, 0], E)
\end{array}\right.
$$

where $A(t)$ is $\omega$-periodic in $t$ and generates an evolution system $(U(t, s))_{t \geq s \geq 0}$ in a Banach space $E$, and $f(t, \cdot, \cdot)$ is $\omega$-periodic in $t$. Hence, the existence of periodic mild solutions using the boundedness and ultimate boundedness of mild solutions when $\omega>r$ is proven.

When operator $A$ in Equation (1.1) satisfies only condition (ii) ( $A$ is non-densely defined), the appropriate solutions to work with will be integral solutions (see Definition 2.1). To our knowledge, we have not seen results about periodic integral solutions, thus we would like to provide one here. Similar to the cases for densely defined operators mentioned above, the most important step here is to show that $P \phi=x_{\omega}(\cdot, \phi)$ is compact, where $x$ is the unique integral solution determined by $\phi$. After this, we are able to apply Horn's fixed point 
theorem and prove under the boundedness and ultimate boundedness assumptions, the existence of $\omega$-periodic integral solutions for Equation (1.1) when $\omega>r$. This way, we can extend the study of periodic solutions for densely defined operators to non-densely defined operators. Finally, we give an example of a partial functional differential equation in continuous functions space with the super-norm.

\section{The Compactness of Operator $P$}

In this section we make the following assumptions:

$\left(H_{1}\right)$ The operator $A$ is a Hille-Yosida operator. That means: there exist $M>0$ and $\tau \in \mathbb{R}$ such that $] \tau, \infty\left[\subset \rho(A)\right.$, and $\sup \left\{(\lambda-\tau)^{n}\left|(\lambda-A)^{-n}\right|: \lambda>\tau\right.$, $n \in N\} \leq M$.

$\left(H_{2}\right)$ The function $F$ is continuous in $t$ and is locally Lipschitz in the second variable in the sense that, for each $h>0$, there exists a constant $k_{1}(h)>0$ such that

$$
\left|F\left(t, \varphi_{1}\right)-F\left(t, \varphi_{2}\right)\right| \leq k_{2}(h)\left|\varphi_{1}-\varphi_{2}\right|, \text { for } t \in[0, h] \text { and }\left|\varphi_{1}\right|,\left|\varphi_{2}\right| \leq h \text {. }
$$

We now list the following definitions.

Definition 2.1: Let $b>0$. A continuous function $x:[-r, b] \rightarrow E$ is called an integral solution of Equation (1.1) if

(i) $\quad \int_{0}^{t} x(s) d s \in D(A)$, for $t \in[0, b]$,

(ii) $\quad x(t)=\varphi(0)+A \int_{0}^{t} x(s) d s+\int_{0}^{t} F\left(s, x_{s}\right) d s$, for $t \in[0, b]$,

(iii) $\quad x_{0}=\varphi$.

Definition 2.2: A continuous function $x:[-r, b] \rightarrow E$ is called a strict solution of Equation (1.1) if

(i) $\quad x \in C^{1}([0, b], E) \cap C([0, b], D(A))$,

(ii) $\quad x$ satisfies Equation (1.1) for $t \in[0, b]$,

(iii) $\quad x_{0}=\varphi$.

Remark 2.1: From the closedness of operator $A$, we see that if an integral solution $x$ of Equation (1.1) is continuous differentiable, then $x$ is a strict solution of Equation (1.1).

We can use methods similar to those in [3] for autonomous equations to obtain the following existence and uniqueness results concerning integral solutions and strict solutions for non-autonomous equations. The details are omitted here.

Theorem 2.1: Assume that $\left(H_{1}\right)$ and $\left(H_{2}\right)$ holds. Then for any $\varphi \in C_{0}=$ $\{\varphi \in C([-r, 0], E): \varphi(0) \in \overline{D(A)}\}$, there exists a unique integral solution $x(\cdot, \varphi)$ of Equation (1.1) with its maximal interval of existence $\left[-r, t_{\varphi}\left[, t_{\varphi}>0\right.\right.$, and

$$
\text { either } t_{\varphi}=\infty \text { or } \limsup _{t \rightarrow t_{\varphi}}|x(t, \varphi)|=\infty
$$

Moreover $x(\cdot, \varphi)$ is a continuous function of $\varphi$ in the sense that, for $\varphi \in C_{0}$ and $t \in\left[0, t_{\varphi}[\right.$, there exist positive constants $L$ and $\epsilon$ such that for any $\psi \in C_{0}$ with $|\varphi-\psi|<\epsilon$, we have

$$
t \in\left[0, t_{\psi}[\text { and }|x(s, \varphi)-x(s, \psi)| \leq L|\varphi-\psi|, s \in[-r, t]\right.
$$

Theorem 2.2: Assume that $\left(H_{1}\right)$ and $\left(H_{2}\right)$ hold. Furthermore, assume that $F$ is continuous differentiable, and for each $h>0$, there exist positive constants $k_{2}(h)$ and $k_{3}(h)$ such that

$$
\left|D_{1} F\left(t, \varphi_{1}\right)-D_{1} F\left(t, \varphi_{2}\right) \leq k_{2}(h)\right| \varphi_{1}-\varphi_{2} \mid
$$




$$
\left|D_{2} F\left(t, \varphi_{1}\right)-D_{2} F\left(t, \varphi_{2}\right)\right| \leq k_{3}(h)\left|\varphi_{1}-\varphi_{2}\right|,
$$

for $t \in[0, h]$ and $\left|\varphi_{1}\right|,\left|\varphi_{2}\right| \leq h$, where $D_{1}$ and $D_{2}$ denote respectively the derivative of $F(t, \varphi)$ with respect to $t$ and $\varphi$. Let $\varphi \in C_{0}$ such that

$$
\varphi^{\prime} \in C_{0}, \varphi(0) \in D(A) \text {, and } \varphi^{\prime}(0)=A \varphi(0)+F(0, \varphi) .
$$

Then the integral solution $x(\varphi)$ of Equation (1.1) is a strict solution in $\left[0, t_{\varphi}[\right.$.

Remark 2.2: If we assume that function $F(t, \varphi)$ is continuous and Lipschitz with respect to the second argument, then the integral solution of Equation (1.1) exists for all $t \geq 0$. Moreover, the solutions $x(t, \varphi)$ are locally bounded in $t$ and $\varphi$. In the following, we are concerned with periodic integral solutions, so we will assume that all integral solutions exist on $[0, \infty)$.

Now, we introduce the part $A_{0}$ of $A$ in $\overline{D(A)}$, defined by

$$
A_{0}=A \text { on } D\left(A_{0}\right)=\{x \in D(A): A x \in \overline{D(A)}\} .
$$

Then it is well known $[1,7]$ that the part $A_{0}$ of $A$ generates a strongly continuous semigroup $T_{0}(\cdot)$ on $\overline{D(A)}$; and for $\varphi \in C_{0}$, the integral solution of Equation (1.1) is given by

$$
x(t)=\left\{\begin{array}{c}
T_{0}(t) \varphi(0)+\lim _{\lambda \rightarrow \infty} \int_{0}^{t} T_{0}(t-s) B_{\lambda} F\left(s, x_{s}\right) d s, \quad t \geq 0, \\
\varphi(t), t \in[-r, 0]
\end{array}\right.
$$

where $B_{\lambda}=\lambda(\lambda-A)^{-1}$; and $x(t) \in \overline{D(A)}, t \geq 0$.

In the sequel, we need to prove the compactness of operator $P$, so we assume:

$\left(H_{3}\right) \quad$ The semigroup $\left(T_{0}(t)\right)_{t \geq 0}$ is compact on $\overline{D(A)}$. That means for each $t>0$, operator $T_{0}(t)$ is compact on $\overline{D(A)}$.

Now we are ready to prove our main result in this paper, that is, the compactness of operator $P$.

Theorem 2.3: Assume that $\left(H_{1}\right),\left(H_{2}\right),\left(H_{3}\right)$ hold and that all integral solutions of Equation (1.1) exist for $t \geq 0$ and are locally bounded (that is, for any $t>0$, the integral solutions of Equation (1.1) are bounded on $[0, t]$ by a constant if their initial functions are bounded by a constant). Let $\omega>r$ be fixed. Then the operator $P \varphi=x_{\omega}(\cdot, \varphi)$ on $C_{0}$ is compact (continuous and takes a bounded set into a precompact set).

Proof: The continuity of $P$ follows from Theorem 2.1. Let $B$ be a bounded set in $C_{0}$. Then, as integral solutions are locally bounded, it follows that the set

$$
W=\left\{x_{\omega}(\cdot, \varphi): \varphi \in B\right\}
$$

is bounded in $C_{0}$. Next, we will show that $W$ is precompact in $C_{0}$ by using Arzela-Ascoli's theorem. To that end, we first prove that for every fixed $\theta \in[-r, 0]$,

$$
\{x(\omega+\theta, \varphi): \varphi \in B\}
$$

is precompact in $\overline{D(A)}$. From (2.1), we have

$$
x(\omega+\theta, \varphi)=T_{0}(\omega+\theta) \varphi(0) \underset{\lambda \rightarrow \infty}{\rightarrow} \int_{0}^{\omega+\theta} T_{0}(\omega+\theta-s) B_{\lambda} F\left(s, x_{s}(\cdot, \varphi)\right) d s .
$$

Since $\omega>r$ is fixed, we can choose $\epsilon>0$ such that $\omega+\theta-\epsilon>0$. Then 


$$
\begin{aligned}
& \int_{0}^{\omega+\theta} T_{0}(\omega+\theta-s) B_{\lambda} F\left(s, x_{s}(\cdot, \varphi)\right) d s \\
= & \int_{0}^{\omega+\theta-\epsilon} T_{0}(\omega+\theta-s) B_{\lambda} F\left(s, x_{s}(\cdot, \varphi)\right) d s \\
+ & \int_{\omega+\theta-\epsilon}^{\omega+\theta} T_{0}(\omega+\theta-s) B_{\lambda} F\left(s, x_{s}(\cdot, \varphi)\right) d s,
\end{aligned}
$$

and

$$
\begin{gathered}
\lim _{\lambda \rightarrow \infty} \int_{0}^{\omega+\theta-\epsilon} T_{0}(\omega+\theta-s) B_{\lambda} F\left(s, x_{s}(\cdot, \varphi)\right) d s \\
=T_{0}(\epsilon) \lim _{\lambda \rightarrow \infty} \int_{0}^{\omega+\theta-\epsilon} T_{0}(\omega+\theta-\epsilon-s) B_{\lambda} F\left(s, x_{s}(\cdot, \varphi)\right) d s .
\end{gathered}
$$

Therefore, by Assumption $\left(H_{3}\right)$ and the local boundedness assumption on integral solutions, we see that

$$
\begin{gathered}
\left\{\lim _{\lambda \rightarrow \infty} \int_{0}^{\omega+\theta-\epsilon} T_{0}(\omega+\theta-s) B_{\lambda} F\left(s, x_{s}(\cdot, \varphi)\right) d s: \varphi \in B\right\} \\
=T_{0}(\epsilon) \quad\left\{\lim _{\lambda \rightarrow \infty} \int_{0}^{\omega+\theta-\epsilon} T_{0}(\omega+\theta-\epsilon-s) B_{\lambda} F\left(s, x_{s}(\cdot, \varphi)\right) d s: \varphi \in B\right\}
\end{gathered}
$$

is precompact in $\overline{D(A)}$ because $T_{0}(\epsilon)$ is applied to a bounded set. Next, for some positive constant $a$,

$$
\begin{gathered}
\lim _{\lambda \rightarrow \infty} \int_{0}^{\omega+\theta} T_{0}(\omega+\theta-s) B_{\lambda} F\left(s, x_{s}(\cdot, \varphi)\right) d s \\
\bar{\lambda} \lim _{\rightarrow \infty} \int_{0}^{\omega+\theta-\epsilon} T_{0}(\omega+\theta-s) B_{\lambda} F\left(s, x_{s}(\cdot, \varphi)\right) d s \mid \\
\leq\left|\lim _{\lambda \rightarrow \infty} \int_{\omega+\theta-\epsilon}^{\omega+\theta} T_{0}(\omega+\theta-s) B_{\lambda} F\left(s, x_{s}(\cdot, \varphi)\right) d s\right| \leq a \epsilon .
\end{gathered}
$$

This implies that the set

$$
\left\{\lim _{\lambda \rightarrow \infty} \int_{0}^{\omega+\theta} T_{0}(\omega+\theta-s) B_{\lambda} F\left(s, x_{s}(\cdot, \varphi)\right) d s: \varphi \in B\right\}
$$

is totally bounded, and therefore the set $\{x(\omega+\theta, \varphi): \varphi \in B\}$ is precompact in $\overline{D(A)}$.

It remains to prove the equicontinuity of functions in set $W$. Let $\theta, \theta_{0} \in[-r, 0]$ with $\theta>\theta_{0}$. Then $\omega+\theta>\omega+\theta_{0}>0$, and

$$
x(\omega+\theta, \varphi)-x\left(\omega+\theta_{0}, \varphi\right)=\left[T_{0}(\omega+\theta)-T_{0}\left(\omega+\theta_{0}\right)\right] \varphi(0)
$$




$$
\begin{aligned}
& +\lim _{\lambda \rightarrow \infty} \int_{0}^{\omega+\theta} T_{0}(\omega+\theta-s) B_{\lambda} F\left(s, x_{s}(\cdot, \varphi)\right) d s \\
& \bar{\lambda} \rightarrow \lim _{0} \int_{0}^{\omega+\theta_{0}} T_{0}\left(\omega+\theta_{0}-s\right) B_{\lambda} F\left(s, x_{s}(\cdot, \varphi)\right) d s .
\end{aligned}
$$

Notice that

$$
\begin{gathered}
\int_{0}^{\omega+\theta} T_{0}(\omega+\theta-s) \\
B_{\lambda} F\left(s, x_{s}(\cdot, \varphi)\right) d s=\int_{0}^{\omega+\theta_{0}} T_{0}(\omega+\theta-s) B_{\lambda} F\left(s, x_{s}(\cdot, \varphi)\right) d s \\
+\int_{\omega+\theta_{0}}^{\omega+\theta} T_{0}(\omega+\theta-s) B_{\lambda} F\left(s, x_{s}(\cdot, \varphi)\right) d s,
\end{gathered}
$$

so we deduce that

$$
\begin{gathered}
\left|x(\omega+\theta, \varphi)-x\left(\omega+\theta_{0}, \varphi\right)\right| \leq\left|T_{0}(\omega+\theta)-T_{0}\left(\omega+\theta_{0}\right)\right||\varphi(0)| \\
+\left|\lim _{\lambda \rightarrow \infty} \int_{0}^{\omega+\theta_{0}}\left[T_{0}(\omega+\theta-s)-T_{0}\left(\omega+\theta_{0}-s\right)\right] B_{\lambda} F\left(s, x_{s}(\cdot, \varphi)\right) d s\right| \\
\quad+\left|\lim _{\lambda \rightarrow \infty} \int_{\omega+\theta_{0}}^{\omega+\theta} T_{0}(\omega+\theta-s) B_{\lambda} F\left(s, x_{s}(\cdot, \varphi)\right) d s\right| .
\end{gathered}
$$

From Assumption $\left(H_{3}\right)$ and a result in [10], semigroup $\left(T_{0}(t)\right)_{t \geq 0}$ is uniformly continuous for $t>0$, which implies that

$$
\lim _{\theta \rightarrow \theta_{0}}\left|T_{0}(\omega+\theta)-T_{0}\left(\omega+\theta_{0}\right)\right|=0
$$

since $\omega+\theta>\omega+\theta_{0}>0$. Furthermore, there exists a positive constant $b$ such that

$$
\left\lfloor\lim _{\lambda \rightarrow \infty} \int_{\omega+\theta_{0}}^{\omega+\theta} T_{0}(\omega+\theta-s) B_{\lambda} F\left(s, x_{s}(\cdot, \varphi)\right) d s \mid \leq b\left(\theta-\theta_{0}\right) .\right.
$$

We also have

$$
\begin{aligned}
& \lambda \underset{\lim }{\lambda \rightarrow \infty} \int_{0}^{\omega+\theta_{0}}\left[T_{0}(\omega+\theta-s)-T_{0}\left(\omega+\theta_{0}-s\right)\right] B_{\lambda} F\left(s, x_{s}(\cdot, \varphi)\right) d s \\
& =\left[T_{0}\left(\theta-\theta_{0}\right)-I\right] \lim _{\lambda \rightarrow \infty} \int_{0}^{\omega+\theta_{0}} T_{0}\left(\omega+\theta_{0}-s\right) B_{\lambda} F\left(s, x_{s}(\cdot, \varphi)\right) d s .
\end{aligned}
$$

As in (2.3), we obtain that there is a compact set $K$ in $\overline{D(A)}$ such that

$$
\lim _{\lambda \rightarrow \infty} \int_{0}^{\omega+\theta_{0}} T_{0}\left(\omega+\theta_{0}-s\right) B_{\lambda} F\left(s, x_{s}(\cdot, \varphi)\right) d s \in K \text { for all } \varphi \in B .
$$

Thus, from Banach-Stenhauss' theorem, we have 


$$
\lim _{\theta \rightarrow \theta_{0}} \sup _{x \in K}\left|\left(T_{0}\left(\theta-\theta_{0}\right)-I\right) x\right|=0
$$

This implies that

$$
\lim _{\theta \rightarrow \theta_{0}^{+}}\left|x(\omega+\theta, \varphi)-x\left(\omega+\theta_{0}, \varphi\right)\right|=0, \text { uniformly for } \varphi \in B .
$$

The proof for $\theta<\theta_{0}$ is similar. This completes the proof.

\section{Boundedness and Periodicity}

After showing the compactness of operator $P$, we can follow $[2,8,14]$ and derive periodic integral solutions for Equation (1.1). In the sequel, we make the following assumption.

$\left(H_{4}\right)$ The function $F(t, \varphi)$ is $\omega$-periodic in $t$ with $\omega>r$.

We also need to bound certain terms involving integral solutions, so we define the following

Definition 3.1: We say that integral solutions of Equation (1.1) are bounded if for each $B_{1}>0$, there is a $B_{2}>0$ such that $|\varphi| \leq B_{1}$ implies $|x(t, \varphi)| \leq B_{2}$ for $t \geq 0$.

Definition 3.2: We say that integral solutions of Equation (1.1) are ultimate bounded if there is a bound $B>0$, such that for each $B_{3}>0$, there is a $k>0$ such that $|\varphi| \leq B_{3}$ and $t \geq k$ imply $|x(t, \varphi)| \leq B$.

We also list the following result as a reference.

Lemma 3.1: [6] (Horn's Fixed Point Theorem) Let $E_{0} \subset E_{1} \subset E_{2}$ be convex subsets of Banach space $Z$, with $E_{0}$ and $E_{2}$ compact subsets and $E_{1}$ open relative to $E_{2}$. Let $P: E_{2} \rightarrow Z$ be a continuous map such that for some integer $m$, one has

$$
\begin{gathered}
P^{j}\left(E_{1}\right) \subset E_{2}, 1 \leq j \leq m-1, \\
P^{j}\left(E_{1}\right) \subset E_{0}, m \leq j \leq 2 m-1,
\end{gathered}
$$

then $P$ has a fixed point in $E_{2}$.

With these preparations, we can prove:

Theorem 3.1: Assume that $\left(H_{1}\right),\left(H_{2}\right),\left(H_{3}\right)$ and $\left(H_{4}\right)$ hold. Furthermore, suppose that integral solutions of Equation (1.1) are bounded and ultimate bounded. Then Equation (1.1) has an w-periodic integral solution.

Proof: From Theorem 2.3, we know that operator $P \phi=x_{\omega}(\cdot, \phi)$ on $C_{0}$ is compact. Let $B$ be the bound in the definition of ultimate boundedness. Then by boundedness, there is a $B_{1}>0$ such that $|\varphi| \leq B$ implies $|x(t, \varphi)| \leq B_{1}$ for $t \geq 0$. Furthermore, there is a $B_{2}>B_{1}$ such that $|\varphi| \leq B_{1}$ implies $|x(t, \varphi)| \leq B_{2}$ for $t \geq 0$. Now, using ultimate boundedness, there is a positive integer $m$ such that $|\varphi| \leq B_{1}$ implies $|x(t, \varphi)| \leq B$ for $t \geq(m-2) \omega$.

Let $x(t)$ be an integral solution of Equation (1.1) and define $y(t)=x(t+\omega)$. Then for $t \geq 0$,

$$
\begin{gathered}
y(t)=x(t+\omega)=T_{0}(t+\omega) \phi(0) \underset{\lambda}{+} \underset{\rightarrow \infty}{\lim _{0} \int^{t+\omega} T_{0}(t+\omega-h) B_{\lambda} F\left(h, x_{h}\right) d h} \\
=T_{0}(t) T_{0}(\omega) \phi(0) \underset{\lambda}{+} \underset{\rightarrow}{\rightarrow} \int_{0}^{\omega} T_{0}(t+\omega-h) B_{\lambda} F\left(h, x_{h}\right) d h
\end{gathered}
$$




$$
\begin{gathered}
+\lim _{\lambda \rightarrow \infty} \int_{\omega}^{t+\omega} T_{0}(t+\omega-h) B_{\lambda} F\left(h, x_{h}\right) d h \\
=T_{0}(t)\left[T_{0}(\omega) \phi(0)+\lim _{\lambda \rightarrow \infty} \int_{0}^{\omega} T_{0}(\omega-h) B_{\lambda} F\left(h, x_{h}\right) d h\right] \\
+\lim _{\lambda \rightarrow \infty} \int_{0}^{t} T_{0}(t-s) B_{\lambda} F\left(s, x_{\omega+s}\right) d s \\
=T_{0}(t) y(0)+\lim _{\lambda \rightarrow \infty} \int_{0}^{t} T_{0}(t-s) B_{\lambda} F\left(s, y_{s}\right) d s .
\end{gathered}
$$

This implies that $y(t)=x(t+\omega)$ is also an integral solution of Equation (1.1). Then the uniqueness implies that $P^{m} \varphi=x_{m \omega}(\cdot, \varphi)$ for $\varphi \in C_{0}$. Thus, we have

$$
\begin{gathered}
\left|P^{j-1} \varphi\right|=\left|x_{(j-1) \omega}(\cdot, \varphi)\right|<B_{2}, j=1,2, \ldots, m-1 \text { and }|\varphi| \leq B_{1}, \\
\left|P^{j-1} \varphi\right|=\left|x_{(j-1) \omega}(\cdot, \varphi)\right|<B, j \geq m \text { and }|\varphi| \leq B_{1} .
\end{gathered}
$$

Let

$$
\begin{gathered}
H=\left\{\varphi \in C_{0}:|\varphi|<B_{2}\right\}, E_{2}=\overline{\operatorname{cov} .(P(H))}, \\
K=\left\{\varphi \in C_{0}:|\varphi|<B_{1}\right\}, E_{1}=K \cap E_{2}, \\
G=\left\{\varphi \in C_{0}:|\varphi|<B\right\}, E_{0}=\overline{\operatorname{cov} \cdot(P(G))},
\end{gathered}
$$

where $\operatorname{cov} .(F)$ is the convex hull of the set $F$. Then we see that $E_{0}, E_{1}$ and $E_{2}$ are convex subsets of $C_{0}$ with $E_{0}$ and $E_{2}$ compact subsets and $E_{1}$ open relative to $E_{2}$ and

$$
\begin{gathered}
P^{j}\left(E_{1}\right) \subset P^{j}(K)=P P^{j-1}(K) \subset P(H) \subset E_{2}, 1 \leq j \leq m-1, \\
P^{j}\left(E_{1}\right) \subset P^{j}(K)=P P^{j-1}(K) \subset P(G) \subset E_{0}, m \leq j \leq 2 m-1 .
\end{gathered}
$$

Consequently, from Horn's fixed point theorem, we know that operator $P$ has a fixed point $\phi$. Then, for the integral solution $x(\cdot)=x(\cdot, \phi)$ with $x_{0}(\cdot, \phi)=\phi$, we see from (3.1) that $y(t)=x(t+\omega, \phi)$ is also an integral solution with $y_{0}=x_{\omega}(\phi)=P \phi=\phi$. Thus the uniqueness implies $x(t, \phi)=y(t)=x(t+\omega, \phi)$; therefore $x(t, \phi)$ is an $\omega$-periodic solution. This completes the proof.

Next, we give a criteria as in [8] to guarantee the boundedness and ultimate boundedness of integral solutions of Equation (1.1) The proof can be found in [8].

Theorem 3.2: Assume that there exist functions $W_{i}, i=1,2,3$ with $W_{i}:[0, \infty[\rightarrow$ $\left[0, \infty\left[, W_{i}(0)=0, W_{i}\right.\right.$ strictly increasing, and $W_{1}(t) \rightarrow \infty$, as $t \rightarrow \infty$. Further assume that there exists a Lyapunov function $V: E \rightarrow \mathbb{R}$ such that for some $M>0$, when $x$ is an integral solution of Equation (1.1) with $|x(t)| \geq M$, then

$$
W_{1}(|x(t)|) \leq V(x(t)) \leq W_{2}(|x(t)|) \text {, and }
$$




$$
\frac{d}{d t} V(x(t)) \leq-W_{3}(|x(t)|) \text { or } V(x(t))-V(x(0)) \leq-\int_{0}^{t} W_{3}(|x(s)|) d s .
$$

Then integral solutions of Equation (1.1) are bounded and ultimate bounded.

\section{An Example}

In the following, we apply our results to the partial functional differential equation

$$
\frac{\partial}{\partial t} u(t, x)=\frac{\partial^{2}}{\partial x^{2}} u(t, x)+f\left(t, x, u_{t}(\cdot, x)\right), u(t, 0)=u(t, 1)=0, t \geq 0, x \in[0,1],
$$

where $u_{t}(\cdot, x)$ (not the partial derivative) is defined by $u_{t}(\cdot, x)(\theta)=u(t+\theta, x)$, $\theta \in[-r, 0], r>0$ is a constant.

We study Equation (4.1) in $E=C[0,1]$, the space of all continuous functions on $[0,1]$ with the super-norm, and define

$$
A u=u^{\prime \prime}, D(A)=\left\{u \in C^{2}[0,1]: u(0)=u(1)=0\right\} .
$$

Then the closure of $D(A)$ is

$$
\overline{D(A)}=C_{0}[0,1]=\{u \in C[0,1]: u(0)=u(1)=0\} \neq C[0,1]=E
$$

thus $A$ is not densely defined on $E$.

Now, (10.2) in [4] is true, and it implies (1.1) in [4]. Thus Hille-Yosida condition $\left(H_{1}\right)$ in our paper is satisfied.

Let $E_{0}$ be the closure of $D(A)$, that is,

$$
E_{0}=\overline{D(A)}=\{u \in C[0,1]: u(0)=u(1)=0\},
$$

and define the part of $A$ as

$$
A_{0} u=A u=u^{\prime \prime}
$$

on the domain

$$
\begin{gathered}
D\left(A_{0}\right)=\left\{u \in D(A): A u \in E_{0}\right\} \\
=\left\{u \in C^{2}[0,1]: u(0)=u(1)=u^{\prime \prime}(0)=u^{\prime \prime}(1)=0\right\} .
\end{gathered}
$$

Lemma 4.1: $A_{0}$ generates a compact semigroup on $E_{0}=\overline{D(A)}$.

Proof: Let $f \in E_{0}$ and consider $\left(\lambda-A_{0}\right) u=f$ for $\lambda \notin(-\infty, 0]$, that is,

$$
\lambda u(t)-u^{\prime \prime}(t)=f(t), u(0)=u(1)=u^{\prime \prime}(0)=u^{\prime \prime}(1)=0 .
$$

From [5], $\lambda u(t)-u^{\prime \prime}(t)=f(t), u(0)=u(1)=0$ has a unique solution $w$ (given on page 372 of [5]). Now, $w \in E_{0}$ and $f \in E_{0}$, so $w^{\prime \prime}=\lambda w-f \in E_{0}$. Thus $w$ is also a unique solution of Equation (4.6). Therefore $\left(\lambda-A_{0}\right)^{-1}$ is well defined on $E_{0}$ for $\lambda \notin(-\infty, 0]$.

Next, similar to the argument in [5], we have

$$
\left\|\left(\lambda-A_{0}\right)^{-1}\right\|_{B\left(E_{0}\right)} \leq \frac{1}{|\lambda| \cos (q / 2)}
$$


where $q=\arg \lambda$ and $B\left(E_{0}\right)$ is the Banach space of all bounded linear operators on $E_{0}$. Now from [4] or [10], $A_{0}$ generates an analytic semigroup on $E_{0}$. Then similar to [10], the analytic semigroup is continuous in the uniform operator topology for $t>0$ and $\left(\lambda-A_{0}\right)^{-1}$, $\lambda \notin(-\infty, 0]$, maps bounded set in $E_{0}$ into bounded set in $E_{0}$ with a uniform bound on their first derivatives. It now follows from Arzela-Ascoli's theorem that $\left(\lambda-A_{0}\right)^{-1}$, $\lambda \notin(-\infty, 0]$ is a compact operator. Therefore, the semigroup is compact according to [10 $\square$

For this example, conditions $\left(H_{1}\right)-\left(H_{4}\right)$ can be met, so that results here can be applied. Details are left to the reader.

\section{Acknowledgements}

The authors would like to thank the referees for their valuable suggestions and comments.

\section{References}

[1] Arendt, A., Vector valued Laplace transforms and Cauchy problems, Israel J. Math. 59 (1987), 327-352.

[2] Amann, H., Periodic solutions of semilinear parabolic equations, Nonl. Anal. A Collection of Papers in Honor of Erich Roth (1978), 1-29.

[3] Adimy, M. and Ezzinbi, K., Local existence and linearized stability for partial functional differential equations, Dyn. Sys. Appl. 7 (1998), 389-404.

[4] Da Prato, G. and Sinestrari, E., Differential operators with nondense domain, Ann. Sc. Norm. Sup. Pisa 14 (1987), 285-344.

[5] Da Prato, G. and Grisvard, P., Sommes doperateurs lineaires et equations differentielles operationnelles, J. Math. Pures Appl. 54 (1975), 305-387.

[6] Hale, J. and Lopes, O., Fixed point theorems and dissipative processes, J. Diff. Eqn. 13 (1966), 391-402.

[7] Kellerman, H. and Hieber, M., Integrated semigroups, J. Funct. Anal. 84 (1989), 160180.

[8] Liu, J., Bounded and periodic solutions of finite delay evolution equations, Nonl. Anal. 34 (1998), 101-111.

[9] Massera, J., The existence of periodic solutions of differential equations, Duke Math. J. 17 (1950), 457-475.

[10] Pazy, A., Semigropus of Linear Operators and Applications to Partial Differential Equations, Springer-Verlag, New York 1983.

[11] Show, S., Remarks on one dimensional delay-differential equations, J. Math. Anal. Appl. 41 (1973), 426-429.

[12] Travis, C. and Webb, G., Existence and stability for partial functional differential equations, Tran. Amer. Math. Soc. 200 (1974), 395-418.

[13] Yoshizawa, T., Stability Theory by Lyapunov's Second Method, Math. Soc. Japan, Tokyo 1966.

[14] Xiang, X. and Ahmed, N., Existence of periodic solutions of semilinear evolution equations with time lags, Nonl. Anal. 11 (1992), 1063-1070. 


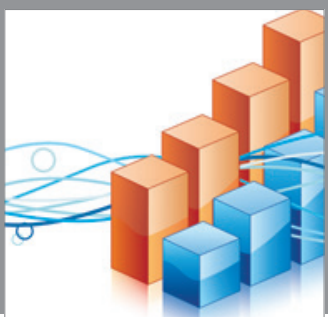

Advances in

Operations Research

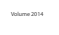

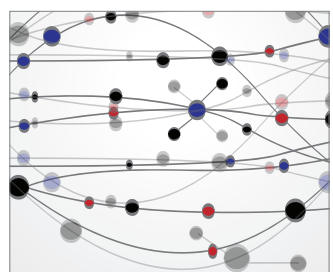

\section{The Scientific} World Journal
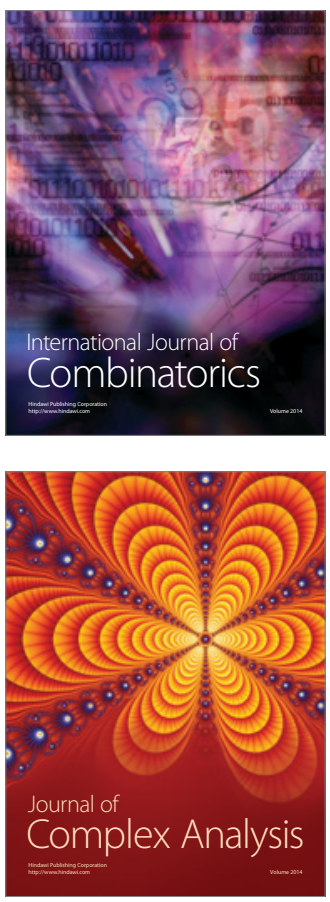

International Journal of

Mathematics and

Mathematical

Sciences
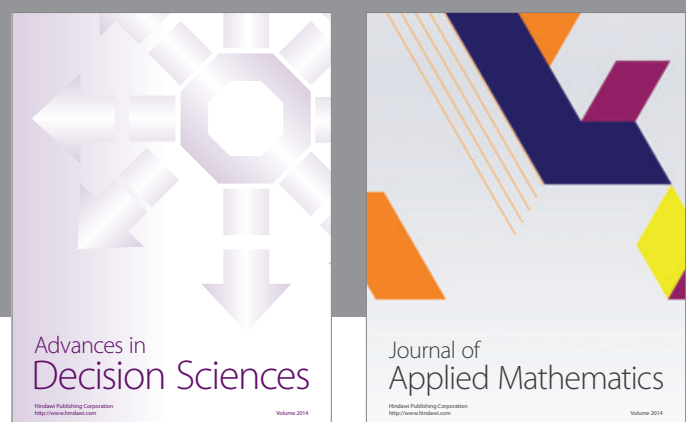

Journal of

Applied Mathematics
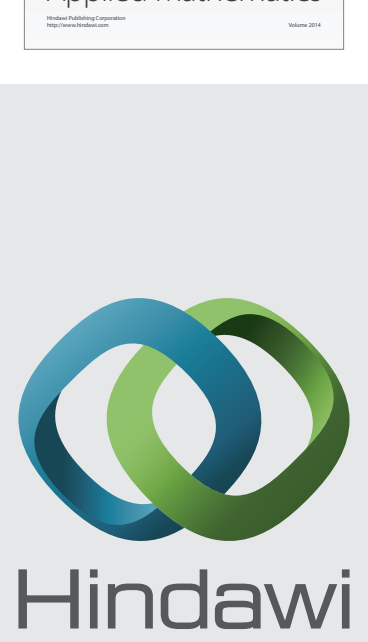

Submit your manuscripts at http://www.hindawi.com
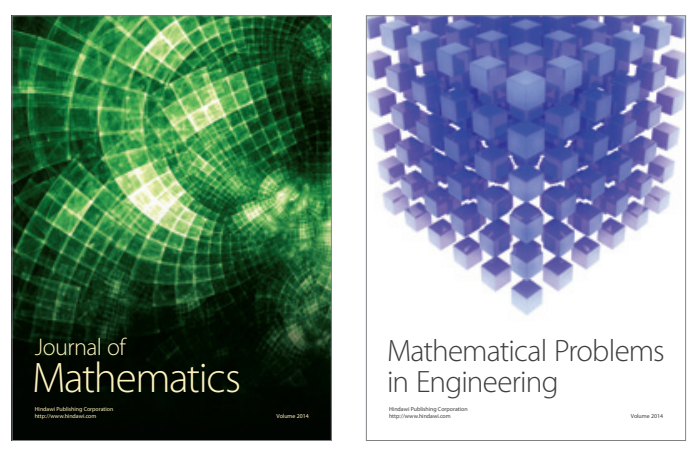

Mathematical Problems in Engineering
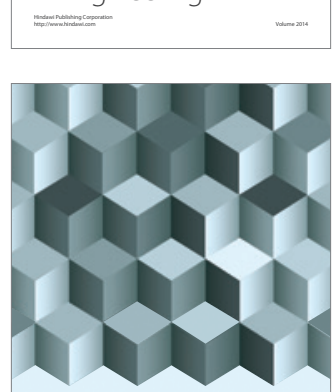

Journal of

Function Spaces
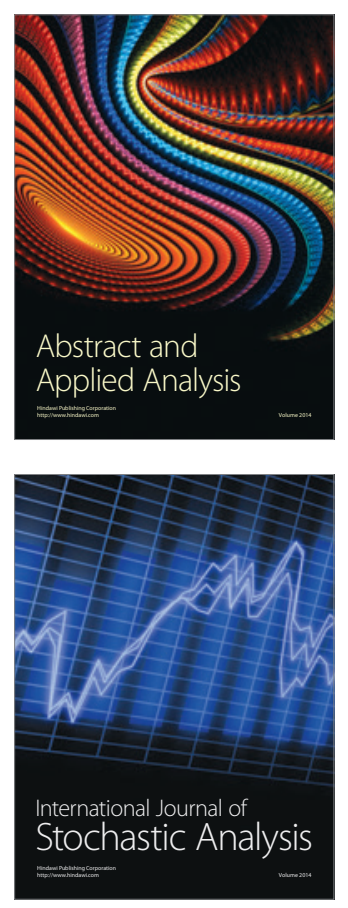

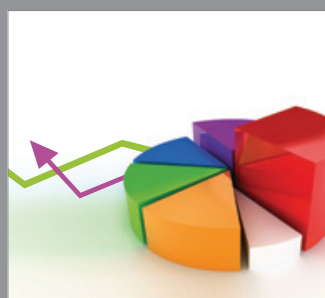

ournal of

Probability and Statistics

Promensencen
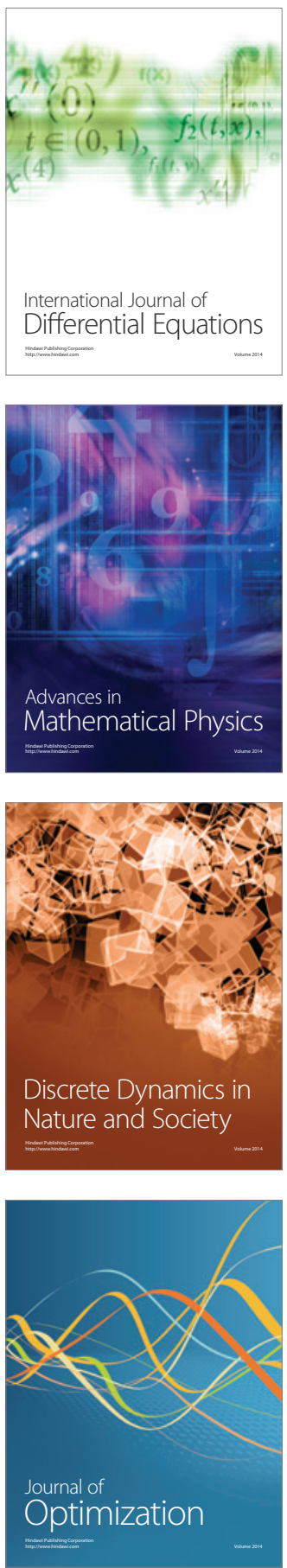